\title{
Predictors for the need for endoscopic therapy in patients with presumed acute upper gastrointesti- nal bleeding
}

Su Sun Kim, Kyung Up Kim, Sung Jun Kim, Seung In Seo, Hyoung Su Kim, Myoung Kuk Jang, Hak Yang Kim, and Woon Geon Shin

Department of Internal Medicine, Hallym University College of Medicine, Chuncheon, Korea

Received: December 8, 2016

Revised : March 3, 2017

Accepted: July 6, 2017

\section{Correspondence to}

Woon Geon Shin, M.D.

Division of Gastroenterology,

Department of Internal Medi-

cine, Hallym University Kang-

dong Sacred Heart Hospital, 150

Seongan-ro, Gangdong-gu, Seoul

05355, Korea

Tel: +82-2-2225-2814

Fax: +82-2-478-6925

E-mail:sgun9139@gmail.com
Background/Aims: Selecting patients with an urgent need for endoscopic hemostasis is difficult based only on simple parameters of presumed acute upper gastrointestinal bleeding. This study assessed easily applicable factors to predict cases in need of urgent endoscopic hemostasis due to acute upper gastrointestinal bleeding.

Methods: The consecutively included patients were divided into the endoscopic hemostasis and nonendoscopic hemostasis groups. We reviewed the enrolled patients' medical records and analyzed various variables and parameters for acute upper gastrointestinal bleeding outcomes such as demographic factors, comorbidities, symptoms, signs, laboratory findings, rebleeding rate, and mortality to evaluate simple predictive factors for endoscopic treatment.

Results: A total of 613 patients were analyzed, including 329 patients in the endoscopic hemostasis and 284 patients in the non-endoscopic hemostasis groups. In the multivariate analysis, a bloody nasogastric lavage (adjusted odds ratio [AOR], 6.786; $95 \%$ confidence interval [CI], 3.990 to 11.543; $p<0.0001$ ) and a hemoglobin level less than $8.6 \mathrm{~g} / \mathrm{dL}$ (AOR, 1.768; 95\% CI, 1.028 to 3.039; $p=0.039$ ) were independent predictors for endoscopic hemostasis. Significant differences in the morbidity rates of endoscopic hemostasis were detected between the group with no predictive factors and the group with one or more predictive factors (OR, 2.677; 95\% CI, 1.920 to $3.733 ; p<0.0001$ ).

Conclusions: A bloody nasogastric lavage and hemoglobin $<8.6 \mathrm{~g} / \mathrm{dL}$ were independent predictors of endoscopic hemostasis in patients with acute upper gastrointestinal bleeding.

Keywords: Gastrointestinal hemorrhage; Hemostasis, endoscopic; Risk factors; Gastric lavage

\section{INTRODUCTION}

Acute upper gastrointestinal bleeding (AUGIB) is an urgent medical situation due to its high morbidity and mortality rates. Despite advances in our medical knowl- edge and equipment technology, the mortality rates of nonvariceal AUGIB remain approximately $3 \%$ to $6 \%[1-3]$ and the mortality rate of variceal bleeding reaches $20 \%$ [4]. Endoscopic hemostasis reduces recurrent bleeding and the surgical treatment rate and improves the sur- 
vival of patients with AUGIB. Therefore, endoscopic intervention has been established as one of the main therapeutic modalities for the management of AUGIB with recent bleeding stigmata [5-8]. However, when and which patients with presumed AUGIB should undertake endoscopy treatment are unclear [9-11]. Some studies have suggested that endoscopy within 12 hours of arrival at the emergency room may be beneficial according to the patients' critical settings [12,13]. Conversely, several studies have reported that emergent endoscopy (within a few hours after presentation) showed no benefits in the mortality of AUGIB patients [5,12,14]. Some guidelines recommend early endoscopy (within 24 hours of presentation) in patients with nonvariceal AUGIB $[8,15,16]$.

Two representative scoring systems are known to be associated with the prognosis, including the rebleeding rate and mortality. The pre-endoscopic Rockall scoring system includes age, pulse rate, systolic blood pressure, and comorbidities [17], whereas the Blatchford scoring system includes presentation with syncope or melena, systolic blood pressure, pulse rate, hepatic disease, cardiac failure, hemoglobin level, and blood urea nitrogen (BUN) [18]. However, the identification of patients who need to undertake urgent endoscopic hemostasis is difficult based on their histories, physical examinations, and simple blood tests without scoring and calculation. Moreover, emergent endoscopy may have many difficulties and dangers, especially during nonregular working hours due to less skilled endoscopy teams and higher fatigue of the endoscopists.

Thus, predicting the patients with presumed AUGIB who require urgent endoscopic hemostasis is very im- portant. For these reasons, we conducted this study to assess easily applicable factors for the prediction of cases in need of an urgent endoscopic procedure prior to endoscopy.

\section{METHODS}

This retrospective study was conducted in Kangdong Sacred Heart Hospital, which is one of five Hallym University medical centers located in Seoul, Republic of Korea. The inclusion criteria were as follows: (1) presumed AUGIB patients (both nonvariceal and variceal bleeding) presenting with hematemesis, melena, and hematochezia within 7 days and (2) at least 15 years of age. The exclusion criteria were as follows: (1) lower gastrointestinal bleeding, (2) gastrointestinal cancer bleeding, and (3) bleeding patients who did not undergo esophagogastroendoscopy.

The consecutively included patients from September 2009 to December 2013 were divided into the endoscopic hemostasis and nonendoscopic hemostasis groups to evaluate simple predictive factors for endoscopic treatment. We defined the endoscopic hemostasis group as those who undergone endoscopic treatment because of spurting, oozing bleeding, or a protruding vessel with nonvariceal bleeding [19]. In case of variceal bleeding, the endoscopic hemostasis group was defined as those who undergone endoscopic treatment because of active bleeding, protruding vessel or white-nipple sign on varix as showed in Fig. 1.

The emergent endoscopy team of our hospital, which consists of one gastroenterologist, one resident train-
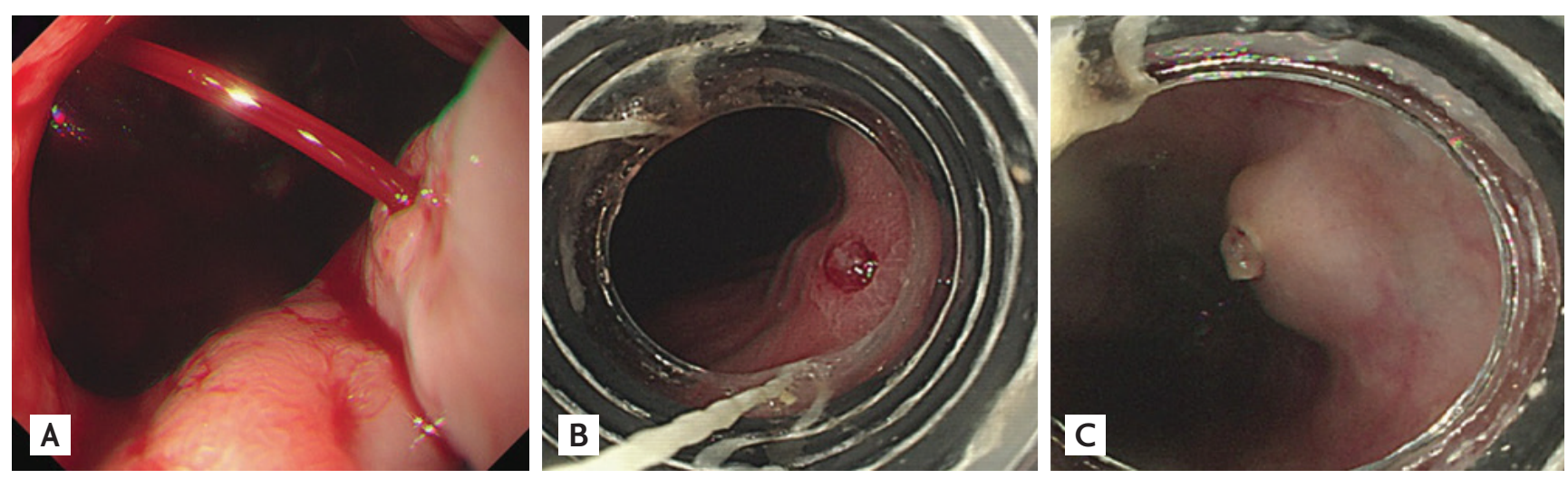

Figure 1. Classification of variceal bleeding requiring an endoscopic hemostasis. (A) Venous spurting from varix, (B) protruding vessel on varix, (C) white-nipple sign on varix. 
ee, and one endoscopy nurse, is on standby for AUGIB control every day. Endoscopic dual hemostasis was performed with epinephrine injection, hemoclipping, and/ or argon plasma coagulation in the endoscopic hemostasis group of nonvariceal bleeding based on the decision of the gastroenterologist performing the endoscopy. One to three sessions of sclerotherapy (histoacryl 1 cc + lipiodol $1 \mathrm{cc} /$ session), or band ligation was performed in the variceal bleeding patients with active bleeding or recent bleeding stigmata. The endoscopic timing was defined as the interval between the hospital visit and starting the endoscopy.

Nasogastric lavage (NGL) was performed at emergency room except the patients who refuse this procedure. About $1 \mathrm{~L}$ saline lavage and drainage was conducted via Levin tube after inserting in stomach. After endoscopic hemostasis, high dose proton pump inhibitor (PPI) continuous infusion (pantoprazole $8 \mathrm{mg} / \mathrm{hr}$ intravenous infusion for 72 hours after $80 \mathrm{mg}$ intravenous loading) was performed. In case of nonendoscopic hemostasis patients underwent daily-standard dose PPI infusion for 24 to 72 hours. In variceal bleeding, 17 times injection of telipressin $1 \mathrm{mg}$ per 4 hours after $2 \mathrm{mg}$ loading and ceftriaxone $2 \mathrm{~g}$ /day for 5 to 7 days injetion was performed regardless of endoscopic hemosasis. Restart of antiplatelet agents or anticoagulants were recommended in 3 to 14 days after initial endoscopy based on the individual risk of rebleeding and thromboembolic event. The patients who had rebleeding was defined as follows: (1) active bleeding during the second-look endoscopy within 48 hours after initial hemostasis, (2) overt hematemesis, (3) passage of fresh blood from the rectum, and (4) a fall in the hemoglobin concentration of $>2 \mathrm{~g} / \mathrm{dL}$ after the initial endoscopic hemostasis. Overall mortality was defined as death of any cause occurring during the hospital stay.

We reviewed the enrolled patients' medical records and analyzed various variables, including comorbidities, medication history, symptoms, vital signs, laboratory findings, and parameters for the outcomes of AUGIB, such as the hospital stay, blood transfusion, surgery, rebleeding rate, and overall mortality.

This study protocol including exemption of informed consent was approved by the Institutional Review Boards of Hallym University Kangdong Sacred Heart Hospital (IRB No:10-091).

\section{Statistical analysis}

The univariate analysis was performed to compare the clinical features or laboratory findings at initial presentation between the endoscopic hemostasis and nonendoscopic hemostasis groups using the chi-square test for categorical variables and the independent sample $t$ test for continuous variables. Multivariate logistic regression analysis was used to assess the independent factors predicting endoscopic hemostasis patients. We used receiver operating characteristic curves to set the cut-off values for the continuous variables. The $p$ values less than 0.05 were considered statistically significant.

All statistical analyses were performed with IBM SPSS for Windows version 19.0 (IBM Corp., Armonk, NY, USA).

\section{RESULTS}

A total of 613 patients were analyzed, including 329 patients in the endoscopic hemostasis group and 284 patients in the nonendoscopic hemostasis group. The baseline characteristics of each group are shown in Table 1. No significant differences in age and gender were observed between the two groups. Regarding comorbidities, liver cirrhosis ( $38 \%$ vs. $20 \%, p<0.0001$ ) and cancer ( $11 \%$ vs. $5 \%, p=0.008$ ) were presented more frequently in the endoscopic hemostasis group than in the nonendoscopic hemostasis group. Interestingly, the proportion of patients using aspirin, other antiplatelet agents, warfarin, or nonsteroidal anti-inflammatory drugs did not differ between the groups (Table 1).

The presence of hematemesis and syncope (including presyncope) as an initial symptom occurred more in the endoscopic hemostasis group than in the nonendoscopic hemostasis group (60\% vs. $47 \%, p=0.002$ and $4 \%$ vs. $1 \%, p=0.037$, respectively). However, other symptoms, such as melena, hematochezia, dizziness, or epigastric pain, showed no significant differences between the two groups. The rate of positive findings in the digital rectal examination was not significantly different between the groups, whereas fresh blood was aspirated from the NGL more frequently in the endoscopic hemostasis group than in the nonendoscopic hemostasis group (126/204 [61.7\%] vs. 40/206 [19.4\%], $p<0.0001)$. The systolic and diastolic blood pressure was significantly lower in the 
Kim SS, et al. Predictors for endoscopic hemostasis

Table 1. Baseline characteristics of the enrolled patients

\begin{tabular}{|c|c|c|c|}
\hline Characteristic & $\begin{array}{l}\text { Endoscopic hemostasis group } \\
\qquad(\mathrm{n}=329)\end{array}$ & $\begin{array}{l}\text { Nonendoscopic hemostasis group } \\
\qquad(\mathrm{n}=284)\end{array}$ & $p$ value \\
\hline Age, yr & $57.1 \pm 13.5$ & $55 \cdot 0 \pm 17.0$ & 0.103 \\
\hline Male sex & $265(81)$ & $215(76)$ & 0.147 \\
\hline \multicolumn{4}{|l|}{ Comorbidity } \\
\hline Liver cirrhosis & $126(38)$ & $58(20)$ & $<0.0001$ \\
\hline Cancer & $37(11)$ & $15(5)$ & 0.008 \\
\hline Ischemic heart disease & $26(8)$ & $22(8)$ & 0.943 \\
\hline Cerebrovascular disease & $27(8)$ & $19(7)$ & 0.177 \\
\hline Chronic kidney disease & $18(6)$ & $14(5)$ & 0.764 \\
\hline Congestive heart failure & $7(2)$ & $5(2)$ & 0.744 \\
\hline History of peptic ulcer & $54(16)$ & $45(16)$ & 0.849 \\
\hline \multicolumn{4}{|l|}{ Medication history } \\
\hline Aspirin & $57(17)$ & $51(18)$ & 0.838 \\
\hline NSAID & $31(9)$ & $28(10)$ & 0.855 \\
\hline Antiplatelet & $20(6)$ & $26(9)$ & 0.149 \\
\hline Warfarin & $14(4)$ & $13(5)$ & 0.846 \\
\hline \multicolumn{4}{|l|}{ Presentation } \\
\hline Melena & $221(67)$ & $203(72)$ & 0.250 \\
\hline Hematemesis & $197(60)$ & $134(47)$ & 0.002 \\
\hline Dizziness & $110(33)$ & $87(31)$ & 0.459 \\
\hline Epigastric pain & $44(13)$ & $43(15)$ & 0.532 \\
\hline Hematochezia & $32(10)$ & $20(7)$ & 0.234 \\
\hline Syncope or presyncope & $14(4)$ & $4(1)$ & 0.037 \\
\hline Rectal examination & 200 & 185 & \\
\hline Gross blood & $166(83)$ & $147(79)$ & 0.373 \\
\hline NGL & 204 & 206 & \\
\hline Bloody & $126(61.7)$ & $40(19.4)$ & $<0.0001$ \\
\hline Systolic BP, mmHg & $101.5 \pm 25.6$ & $110.8 \pm 22.0$ & $<0.0001$ \\
\hline Diastolic BP, mmHg & $61.7 \pm 21.2$ & $69.7 \pm 15.9$ & $<0.0001$ \\
\hline Heart rate, beats/min & $94 \cdot 3 \pm 21.3$ & $92.0 \pm 18.9$ & 0.152 \\
\hline Hemoglobin at presentation, $g / d L$ & $8.9 \pm 2.7$ & $9.9 \pm 3.2$ & $<0.0001$ \\
\hline Platelet count, $\times 10^{3} / \mu \mathrm{L}$ & $190.0 \pm 103.4$ & $240.2 \pm 126.8$ & $<0.0001$ \\
\hline INR & $1.4 \pm 1.0$ & $1.3 \pm 0.9$ & 0.019 \\
\hline BUN, mg/dL & $37.0 \pm 23.1$ & $33.1 \pm 23.4$ & 0.037 \\
\hline Creatinine, mg/dL & $1.5 \pm 1.7$ & $1.3 \pm 1.5$ & 0.189 \\
\hline Timing, hr & $2.33(0.3-67)$ & $3.00(0.2-96)$ & 0.010 \\
\hline \multicolumn{4}{|l|}{ Bleeding cause } \\
\hline Varix & $138(42)$ & $44(16)$ & $<0.0001$ \\
\hline Gastric ulcer & $111(34)$ & $111(39)$ & 0.170 \\
\hline Duodenal ulcer & $47(14)$ & $52(18)$ & 0.177 \\
\hline Mallory-Weiss & $22(7)$ & $36(13)$ & 0.180 \\
\hline Others & $11(3)$ & $41(14)$ & 0.120 \\
\hline
\end{tabular}

Values are presented as mean $\pm \mathrm{SD}$, number (\%), or mean (range).

NSAID, nonsteroidal anti-inflammatory drug; NGL, nasogastric lavage; BP, blood pressure; INR, international normalized ratio; BUN, blood urea nitrogen. 
endoscopic hemostasis group than in the nonendoscopic hemostasis group $(101.5 \pm 25.6 \mathrm{mmHg}$ vs. $110.8 \pm 22.0$ $\mathrm{mmHg}, p<0.0001$ and $61.7 \pm 21.2 \mathrm{mmHg}$ vs. $69.7 \pm 15.9$ $\mathrm{mmHg}, p<0.0001$, respectively). However, the heart rate was not significantly different between the groups.

In the laboratory findings at presentation, the hemoglobin $(8.9 \pm 2.7 \mathrm{~g} / \mathrm{dL}$ vs. $9.9 \pm 3.2 \mathrm{~g} / \mathrm{dL}, p<0.0001)$, platelet count $\left(190.0 \pm 103.4 \times 10^{3}\right.$ vs. $\left.240.2 \pm 126.8 \times 10^{3}, p<0.0001\right)$, international normalized ratio $(1.4 \pm 1.0$ vs. $1.3 \pm 0.9, p=$ 0.019), and BUN (37.0 \pm 23.1 vs. $33.1 \pm 23.4, p=0.037)$ were significantly different between the groups. The endoscopic timing for the endoscopic hemostasis group was shorter than the timing for the nonendoscopic hemostasis group (3.9 \pm 6.6 hours vs. $5.7 \pm 10.1$ hours, $p=0.010$ ).

The endoscopic diagnoses of the included patients are shown in Table 1. A total of 321 patients $(52.4 \%)$ had a peptic ulcer, 182 patients (29.7\%) had varix, 58 patients (9.5\%) had Mallory-Weiss tearing, and 52 patients (8.4\%) had other diagnoses, such as hemorrhagic gastritis and angiodysplasia. Variceal bleeding was present at a higher proportion in the endoscopic hemostasis group than in the nonendoscopic hemostasis group (42\% vs. $16 \%$, p $<0.0001$ ), whereas the other diagnoses did not significantly differ between the groups.

The outcomes of patients with AUGIB are shown in Table 2. A total of 65 patients (11\%) had rebleeding, including 46 in the endoscopic hemostasis group and 19 in the nonendoscopic hemostasis group ( $14 \%$ vs. $7 \%, p$ $=0.003$ ). The number of transfusions within 24 hours was significantly different between the groups $(3.4 \pm 2.9$ units vs. $2.0 \pm 1.9$ units, $p<0.0001$ ). The lengths of the hospital stays were also longer in the endoscopic hemostasis group ( $11.3 \pm 9.5$ days vs. 9.7 \pm 9.7 days, $p=0.033)$. In our series, 56 patients (9.1\%) eventually died at the hospital, including 38 in the endoscopic hemostasis group
(27 with varix bleeding and 11 with peptic ulcer bleeding) and 18 in the nonendoscopic hemostasis group (one with pneumonia, six with sepsis, four with cancer progression, six with cardiovascular disease, and one with pulmonary alveolar hemorrhage) ( $12 \%$ vs. $6 \%, p=0.026$ ).

The risk factors associated with endoscopic hemostasis in the univariate analysis were used to perform the multivariate analysis. The results of multivariate analysis for independent predictive variables of endoscopic hemostasisis were shown in Table 3. A bloody NGL (adjusted odds ratio [AOR], 6.786; 95\% confidence interval [CI], 3.990 to 11.543; $p<0.0001$ ) and hemoglobin $<8.6 \mathrm{~g} /$ dL (AOR, 1.768; 95\% CI, 1.028 to 3.039; $p=0.039$ ) were independent predictors of endoscopic hemostasis.

When a hemoglobin cut-off value of $<8.6 \mathrm{~g} / \mathrm{dL}$ was used, the sensitivity was $47 \%$, the specificity was $63 \%$, the positive predictive value (PPV) was $59 \%$, and the negative predictive value (NPV) was 51\%. A bloody NGL, which was another independent factor for endoscopic hemostasis, showed a sensitivity of $62 \%$, specificity of $81 \%$, PPV of $76 \%$, and NPV of $68 \%$ (Table 4 ).

To determine how well these variables predicted the need of endoscopic hemostasis, we developed predictive groups based on the number of predictive factors (bloody NGL and hemoglobin < $8.6 \mathrm{~g} / \mathrm{dL}$ ). When no predictors were present, 39.3\% (97/247) of the patients underwent endoscopic hemostasis. A significant difference in the morbidity rates of endoscopic hemostasis group were observed between the group with no predictive factors and the group with one or more predictive factors (OR, 2.677; 95\% CI, 1.920 to 3.733; $p<0.0001$ ) (Table 5). The percentage of patients with endoscopic hemostasis increased with the number of predictive factors $(59.9 \%$ [184/307] and $81.4 \%$ [48/59] in the one to two predictive factor group, $p<0.0001)$.

Table 2. The outcomes of patients with acute upper gastrointestinal bleeding

\begin{tabular}{|c|c|c|c|}
\hline & $\begin{array}{c}\text { Endoscopic } \\
\text { hemostasis group }(n=329)\end{array}$ & $\begin{array}{c}\text { Nonendoscopic } \\
\text { hemostasis group }(n=284)\end{array}$ & $p$ value \\
\hline Transfusion requirements within $24 \mathrm{hr}$, unit & $3.4 \pm 2.9$ & $2.0 \pm 1.9$ & $<0.0001$ \\
\hline Rebleeding rate & $46(14)$ & $19(7)$ & 0.003 \\
\hline Surgery & $7(2)$ & $3(1)$ & 0.296 \\
\hline Hospital stay, day & $11.3 \pm 9.5$ & $9.7 \pm 9.7$ & 0.033 \\
\hline In-hospital mortality & $38(12)$ & $18(6)$ & 0.026 \\
\hline
\end{tabular}

Values are presented as mean \pm SD or number (\%). 
Table 3. The multivariate analysis of risk factors for endoscopic hemostasis

\begin{tabular}{lccc}
\hline Factor & Odds ratio & $95 \%$ CI & $p$ value \\
\hline Bloody NGL & 6.786 & $3.990-11.543$ & $<0.0001$ \\
Hemoglobin $(<8.6 \mathrm{~g} / \mathrm{dL})$ & 1.768 & $1.028-3.039$ & 0.039 \\
Platelet count $\left(<175 \times 10^{3} / \mu \mathrm{L}\right)$ & 1.448 & $0.783-2.678$ & 0.238 \\
Liver cirrhosis & 0.923 & $0.428-1.994$ & 0.839 \\
Cancer & 2.590 & $0.852-7.877$ & 0.093 \\
Hematemesis & 0.831 & $0.487-1.417$ & 0.497 \\
Syncope or presyncope & 1.026 & $0.267-3.939$ & 0.970 \\
Systolic BP & 0.997 & $0.977-1.017$ & 0.764 \\
Diastolic BP & 0.983 & $0.955-1.011$ & 0.418 \\
INR & 1.000 & $0.758-1.320$ & 0.227 \\
BUN & 1.008 & $0.998-1.017$ & 0.115 \\
Endoscopic timing & 0.966 & $0.930-1.002$ & 0.067 \\
\hline CI
\end{tabular}

CI, confidence interval; NGL, nasogastric lavage; BP, blood pressure; INR, international normalized ratio; BUN, blood urea nitrogen.

Table 4. Sensitivity, specificity, and positive and negative predictive values of the risk factors for endoscopic hemostasis in AUGIB patients

\begin{tabular}{lcccc}
\hline & $\begin{array}{c}\text { Sensitivity, } \\
\%\end{array}$ & $\begin{array}{c}\text { Specificity, } \\
\%\end{array}$ & $\begin{array}{c}\text { PPV, } \\
\%\end{array}$ & $\begin{array}{c}\text { NPV, } \\
\%\end{array}$ \\
\hline Hemoglobin & 47 & 63 & 59 & 51 \\
Bloody NGL & 62 & 81 & 76 & 68 \\
\hline
\end{tabular}

AUGIB, acute upper gastrointestinal bleeding; PPV, positive predictive value; NPV, negative predictive value; NGL, nasogastric lavage.

Table 5. Model for predicting endoscopic hemostasis according to the presence of risk factors and the number of risk factors

\begin{tabular}{lr}
\hline Risk factor & Endoscopic hemostasis events \\
\hline Risk factors present & $97 / 247(39.3)$ \\
No & $232 / 366(63.4)^{\mathrm{a}}$ \\
Yes & \\
No. of risk factors ${ }^{\mathrm{b}}$ & $97 / 247(39.3)$ \\
O & $184 / 307(59.9)$ \\
1 & $48 / 59(81.4)$ \\
\hline
\end{tabular}

Values are presented as number/total number (\%).

${ }^{a}$ Odds ratio, 2.677; 95\% confidence interval, 1.920-3.733; $p<$ 0.0001 .

${ }^{b} p<0.0001$.

\section{DISCUSSION}

We cannot diagnose the exact causes of presumed AUGIB prior to endoscopy. The decision concerning the optimal timing to perform an endoscopy in patients with AUGIB is one the most important concerns after medical resuscitation. Thus, this study focused on predictors that could easily predict ongoing AUGIB, which is needed to prompt endoscopic hemostasis in a preendoscopy setting. The independent predictors of endoscopic hemostasis were a bloody NGL and hemoglobin less than $8.6 \mathrm{~g} / \mathrm{dL}$. The OR of endoscopic hemostasis was 2.677 in patients who had these predictors compared with patients who had no predictors. Importantly, the presence of more predictors in AUGIB patients indicated an increased risk of endoscopic hemostasis.

The good PPV (76\%) of a bloody NGL can be interpreted that patients with fresh-blood drainage from a NGL need to undergo prompt endoscopic hemostasis. Some previous studies reported that a bloody NGL could predict high-risk bleeding stigmata or a high rebleeding rate [13,20-22]. For example, one study documented that $45 \%$ of patients with a bloody NGL compared to only $15 \%$ of patients with a clear NGL had high-risk bleeding stigmata on endoscopy [20]. A recent meta-analysis reported that an NGL with red blood increased the likelihood (likelihood ratio, 3.1; 95\% CI, 1.2 to 14.0) of a severe AUGIB requiring urgent intervention [23]. Therefore, an 
international consensus has recommended considering NGL in selected patients for a pre-endoscopy workup due to its possible prognostic value [8]. However, Korean guideline [16] reported that NGL has limitation because NGL is an uncomfortable procedure for patients, has a high false-negative rate, and does not reduce the mortality rate [24].

A low hemoglobin level has been reported as one of the predictors for urgent endoscopy in previous studies $[23,25,26]$. A recent meta-analysis reported that the likelihood ratio of severe AUGIB ranged between 4.5 and 6.2, with a cut-off hemoglobin level of $8 \mathrm{~g} / \mathrm{dL}$ [23]. One study with 3,386 patients with bleeding peptic ulcers after initial hemostasis reported that a hemoglobin level $<10 \mathrm{~g} / \mathrm{dL}$ was one predictive values for rebleeding (OR, 1.87; 95\% CI, 1.18 to 2.96 ) [25].

Two typical risk scoring systems (the pre-endoscopy Rockall and Blatchford scores) are used to predict the prognosis of AUGIB prior to the index endoscopy. However, neither the pre-endoscopy Rockall score nor the Blatchford score were useful for predicting the need for endoscopic therapy. The Blatchford score had a role only in identifying patients who might not require endoscopic hemostasis [27]. Furthermore, neither of these scoring systems could predict rebleeding [1].

Our study has some strengths. We included detailed demographic factors, comorbidities, symptoms, signs, and laboratory findings to better adjust for potential confounders. Second, our predictors can be acquired within 1 hour without calculation of prognostic scales in the emergency room in patients with not only nonvariceal bleeding but also with variceal bleeding. This ability would enable prompt decision making for urgent endoscopy.

This study also has some weaknesses. First, inevitable selection bias may be present. However, we enrolled AUGIB patients consecutively to minimize this selection bias, and all patients underwent endoscopy as soon as possible if their vital signs were maintained without active infection. Indeed, our study demonstrated that the endoscopic hemostasis group had a significantly worse prognosis based on the transfusion requirements within 24 hours, rebleeding rate, duration of hospital stay, and in-hospital all-cause mortality (Table 4), which was similar to the findings in previous studies [5,28]. Therefore, we believe that our predictors could be meaningful, reliable, and clinically applicable factors despite selection bias, although a prospective and randomized study is warranted to confirm the usefulness of NGL and hemoglobin in patients with AUGIB. Second, we may have another bias from the differences of the gastroenterologists' skill and relatively small sample size for analyzing retrospective data.

In conclusion, a fresh bloody NGL and hemoglobin < $8.6 \mathrm{~g} / \mathrm{dL}$ might be useful, easily applicable, and independent predictors for endoscopic hemostasis in patients with AUGIB.

\section{KEY MESSAGE}

1. A fresh bloody nasogastric lavage and hemoglobin $<8.6 \mathrm{~g} / \mathrm{dL}$ might be useful, easily applicable, and independent predictors for endoscopic hemostasis in a pre-endoscopy setting.

2. The odds ratio of endoscopic hemostasis was 2.677 in patients who had these predictors compared with patients who had no predictors.

3. The presence of more predictors indicated an increased risk of endoscopic hemostasis.

\section{Conflict of interest}

No potential conflict of interest relevant to this article was reported.

\section{REFERENCES}

1. Yang HM, Jeon SW, Jung JT, et al. Comparison of scoring systems for nonvariceal upper gastrointestinal bleeding: a multicenter prospective cohort study. J Gastroenterol Hepatol 2016;31:119-125.

2. Aquarius M, Smeets FG, Konijn HW, et al. Prospective multicenter validation of the Glasgow Blatchford bleeding score in the management of patients with upper gastrointestinal hemorrhage presenting at an emergency department. Eur J Gastroenterol Hepatol 2015;27:1011-1016.

3. Tsoi KK, Chiu PW, Chan FK, Ching JY, Lau JY, Sung JJ. The risk of peptic ulcer bleeding mortality in relation to hospital admission on holidays: a cohort study on 8,222 cases of peptic ulcer bleeding. Am J Gastroenterol 2012; 107:405-410. 
4. Garbuzenko DV. Current approaches to the management of patients with liver cirrhosis who have acute esophageal variceal bleeding. Curr Med Res Opin 2016;32:467-475.

5. Hearnshaw SA, Logan RF, Lowe D, Travis SP, Murphy MF, Palmer KR. Use of endoscopy for management of acute upper gastrointestinal bleeding in the UK: results of a nationwide audit. Gut 2010;59:1022-1029.

6. Sacks HS, Chalmers TC, Blum AL, Berrier J, Pagano D. Endoscopic hemostasis: an effective therapy for bleeding peptic ulcers. JAMA 1990;264:494-499.

7. Cook DJ, Guyatt GH, Salena BJ, Laine LA. Endoscopic therapy for acute nonvariceal upper gastrointestinal hemorrhage: a meta-analysis. Gastroenterology 1992;102:139-148.

8. Barkun AN, Bardou M, Kuipers EJ, et al. International consensus recommendations on the management of patients with nonvariceal upper gastrointestinal bleeding. Ann Intern Med 2010;152:101-113.

9. Cho HS, Han DS, Ahn SB, et al. Comparison of the effectiveness of interventional endoscopy in bleeding peptic ulcer disease according to the timing of endoscopy. Gut Liver 2009;3:266-270.

10. Tsoi KK, Ma TK, Sung JJ. Endoscopy for upper gastrointestinal bleeding: how urgent is it? Nat Rev Gastroenterol Hepatol 2009;6:463-469.

11. Laine L, Cohen H, Brodhead J, Cantor D, Garcia F, Mosquera M. Prospective evaluation of immediate versus delayed refeeding and prognostic value of endoscopy in patients with upper gastrointestinal hemorrhage. Gastroenterology 1992;102:314-316.

12. Lin HJ, Wang K, Perng CL, et al. Early or delayed endoscopy for patients with peptic ulcer bleeding: a prospective randomized study. J Clin Gastroenterol 1996;22:267-271.

13. Adamopoulos AB, Baibas NM, Efstathiou SP, et al. Differentiation between patients with acute upper gastrointestinal bleeding who need early urgent upper gastrointestinal endoscopy and those who do not: a prospective study. Eur J Gastroenterol Hepatol 2003;15:381-387.

14. Schacher GM, Lesbros-Pantoflickova D, Ortner MA, Wasserfallen JB, Blum AL, Dorta G. Is early endoscopy in the emergency room beneficial in patients with bleeding peptic ulcer? A "fortuitously controlled" study. Endoscopy 2005;37:324-328.

15. Hwang JH, Fisher DA, Ben-Menachem T, et al. The role of endoscopy in the management of acute non-variceal upper GI bleeding. Gastrointest Endosc 2012;75:1132-1138.
16. Chung IK, Lee DH, Kim HU, et al. Guidelines of treatment for bleeding peptic ulcer disease. Korean J Gastroenterol 2009;54:298-308.

17. Rockall TA, Logan RF, Devlin HB, Northfield TC. Risk assessment after acute upper gastrointestinal haemorrhage. Gut 1996;38:316-321.

18. Blatchford O, Murray WR, Blatchford M. A risk score to predict need for treatment for upper-gastrointestinal haemorrhage. Lancet 2000;356:1318-1321.

19. Forrest JA, Finlayson ND, Shearman DJ. Endoscopy in gastrointestinal bleeding. Lancet 1974;2:394-397.

20. Aljebreen AM, Fallone CA, Barkun AN. Nasogastric aspirate predicts high-risk endoscopic lesions in patients with acute upper-GI bleeding. Gastrointest Endosc 2004; 59:172-178.

21. Perng CL, Lin HJ, Chen CJ, Lee FY, Lee SD, Lee CH. Characteristics of patients with bleeding peptic ulcer requiring emergency endoscopy and aggressive treatment. Am J Gastroenterol 1994;89:1811-1814.

22. Huang ES, Karsan S, Kanwal F, Singh I, Makhani M, Spiegel BM. Impact of nasogastric lavage on outcomes in acute GI bleeding. Gastrointest Endosc 2011;74:971-980.

23. Srygley FD, Gerardo CJ, Tran T, Fisher DA. Does this patient have a severe upper gastrointestinal bleed? JAMA 2012;307:1072-1079.

24. Pallin DJ, Saltzman JR. Is nasogastric tube lavage in patients with acute upper GI bleeding indicated or antiquated? Gastrointest Endosc 2011;74:981-984.

25. Wong SK, Yu LM, Lau JY, et al. Prediction of therapeutic failure after adrenaline injection plus heater probe treatment in patients with bleeding peptic ulcer. Gut 2002;50: 322-325.

26. Tomizawa M, Shinozaki F, Hasegawa R, et al. Reduced hemoglobin and increased C-reactive protein are associated with upper gastrointestinal bleeding. World J Gastroenterol 2014;20:1311-1317.

27. Pang SH, Ching JY, Lau JY, Sung JJ, Graham DY, Chan FK. Comparing the Blatchford and pre-endoscopic Rockall score in predicting the need for endoscopic therapy in patients with upper GI hemorrhage. Gastrointest Endosc 2010;71:1134-1140.

28. Laine L, McQuaid KR. Endoscopic therapy for bleeding ulcers: an evidence-based approach based on meta-analyses of randomized controlled trials. Clin Gastroenterol Hepatol 2009;7:33-47. 